\title{
Der Nordpol als Aufmerksamkeitsmagnet
}

Neue Monographien zur Konjunktur der Arktis im 19. und 20. Jahrhundert

Christian Holtorf

Cavell, Janice, 2008. Tracing the Connected Narrative. Arctic Exploration in British Print Culture, 1818-1860, Toronto: University of Toronto Press, geb. 352 S., 57,99 €/60 \$, ISBN-13: 978-0-8020-9280-9.

Potter, Russel A., 2007. Arctic Spectacles. The Frozen North in Visual Culture, 1818-1875, Seattle: University of Washington Press, geb. 272 S., 32 Abb., 50 \$, ISBN-13: 978-0-295-98679-1.

Robinson, Michael F., 2006. The Coldest Crucible. Arctic Exploration and American Culture, Chicago: The University of Chicago Press, geb. 20 S., 14 Abb., 39 \$, ISBN-13: 978-0-226-72184-2.

Wamsley, Douglas W., 2009. Polar Hayes. The Life and Contributions of Isaac Israel Hayes, M.D., Philadelphia: American Philosophical Society, geb. xvi + 574 S., 75 \$, ISBN-13: 978-0-87169-262-7.

Während des vierten Internationalen Polarjahres, das den Zeitraum zwischen März 2007 und März 2009 umfasste, wurde häufig darauf hingewiesen, dass Polarjahre vom Internationalen Wissenschaftsrat mit einer bestimmten Regelmäßigkeit ausgerufen werden. Vor 125 Jahren fand das erste Internationale Polarjahr statt (1882/83), ein halbes Jahrhundert später das zweite (1932/33) und weitere 25 Jahre später das Internationale Geophysikalische Jahr (1957/58). Dieses Mal fiel die Wissenschaftsinitiative mit der hundertsten Wiederkehr der beiden spektakulären, aber vielfach bezweifelten Entdeckungen des Nordpols durch Frederick Cook (1908) und Robert Peary (1909) 
zusammen. Nicht zuletzt aufgrund dieser Häufung runder Jubiläen sind eine Reihe von Darstellungen erschienen, in denen die Geschichte der Arktisexpeditionen noch einmal neu erzählt wird (McGoogan 2008, Oeser 2008, Sawin 2009, Zeilinger 2009, vgl. Felsch 2009).

\section{Medien der Imagination}

Doch es war gar nicht erst nötig, das öffentliche Interesse auf die Arktis zu lenken. „In fact Arctic fever still rages today [...]. It persists even within scholarly studies of exploration", betont der amerikanische Wissenschaftshistoriker Michael F. Robinson in The Coldest Crucible. Arctic Exploration and American Culture (S. 163). Die Jahrestage zeigen nämlich, dass die Arktisforschung auch im 19. und 20 Jahrhundert schon gleichermaßen im Fokus der Wissenschaft und Licht der Öffentlichkeit standen. Zwar stilisierte Robert Peary (1856-1920) seine Polarfahrten als Rückkehr zu einer ursprünglichen Natur und Kritik an der industrialisierten Gesellschaft, doch „arctic expeditions", so Robinson, ,were not the lonely affairs that Peary expected“ (S. 1). Peary war vielmehr selber ein Medienprofi, der es verstand, sich ins Rampenlicht der Nation zu stellen. Indem er die Arktis als romantische Gegenwelt entwarf, erschloss er finanzielle Ressourcen: Er verkaufte die Rechte seiner Texte, verdiente Honorare für öffentliche Vorträge und sicherte sich dabei stets die Deutungshoheit über die Ergebnisse seiner Reisen. Wer die Arktis für ein Refugium von Helden hält, die sich selbst und die Natur überwinden, um triumphal zurückzukehren, reproduziert ein Bild, das Abenteurer wie Robert Peary selbst entworfen haben. Die hier vorzustellenden amerikanischen und britischen Neuerscheinungen zur Polargeschichte reflektieren dieses Wechselverhältnis von verschiedenen Seiten. Sie untersuchen den Einfluss neuer Medien und Technologien auf die Geschichte der öffentlichen Wahrnehmung der Nordpolforschung und zeigen, wie mediale Öffentlichkeiten Einfluss auf die Wissenschaft nahmen (vgl. Borck 2009).

Dass Arktis-Imaginationen Maler und Schriftsteller inspirierten und in Museen, Ausstellungen und Vortragssälen inszeniert wurden, haben Untersuchungen von Robert David (2000) oder Francis Spufford (1997) bereits gezeigt. Am Nordpol wurden aber nicht nur neue Nationalhelden geboren, sondern dort spiegelten sich auch vielfältige kulturelle Entwicklungen in deren Heimatstaaten, so Robinsons zentrale These. Tatsächlich hatte der zweite Sekretär der britischen Admiralität, John Barrow, die Arktis in den 1820er Jahren als „new theater of war“ (Robinson, S. 22) neu erfunden, um eine Aufgabe für seine beschäftigungslose Marine zu schaffen. Robinson fragt deshalb, welche Erwartungen den Expeditionen jeweils zu Grunde lagen, welche Leistungen erwartet wurden, welcher Aufwand gerechtfertigt schien und wie die Ergebnisse vermittelt werden sollten. 
Die Öffentlichkeit war nämlich keineswegs ohne Weiteres für Expeditionen zum Nordpol zu gewinnen, da doch, so Robinson, trotz hoher Risiken kein unmittelbarer Nutzen zu erwarten war. Erst Journalisten wie James Gordon Bennett vom New York Herald machten die Polarforscher zu Helden, die auf öffentliches Interesse stießen und nebenbei auch die Nachfrage nach Zeitungen förderten: „Stories, more than specimens or scientific observations, constituted the real currency of Arctic exploration." (Robinson 2006: 6) Anschließend an eine Untersuchung von Riffenburgh (1993), der gezeigt hat, wie stark sich britische und amerikanische Presse, Sensationslust und geografische Entdeckungen sowohl in der Arktis als auch in Afrika zwischen 1855 und 1910 gegenseitig gefördert haben, wählt Robinson das Beispiel des amerikanischen Arztes und Abenteurers Elisha Kent Kane (1820-1857). Kane begeisterte zwischen 1851 und 1853 die Vereinigten Staaten, weil er Draufgängertum mit der schon mittelalterlichen Fantasie eines eisfreien Polarmeeres verband, das von einer Eisbarriere umschlossen sei. Dort könnte der seit 1845 verschollene britische Offizier John Franklin eingeschlossen sein, dessen Verschwinden eine spektakuläre internationale Suchaktion ausgelöst hatte. Der New Yorker Millionär Henry Grinnell finanzierte Kanes Expedition, aber die Ergebnisse dieser Reise waren mager. Doch dass zwei Expeditionsmitglieder das offene Polarmeer gesichtet haben wollten, verbreitete Kane so wirkungsvoll, dass er zur nationalen Berühmtheit und moralischen Instanz für die amerikanische Mittelschicht aufstieg. Spätere Expeditionsleiter, so Robinson, behaupteten erst gar keinen wissenschaftlichen Gewinn mehr, sondern setzten von vornherein auf ,danger, tragedy, and scandal“" (S. 105). Standen die Polarforscher der 1850er und 1860er Jahre noch für wissenschaftlichen und technologischen Fortschritt, waren ihre Nachfolger am Ende des Jahrhunderts demgegenüber zu Repräsentanten einer glorreichen Vergangenheit geworden, die das Publikum gerade durch die Zurückweisung der Annehmlichkeiten der Zivilisation fesselten (S. 161).

\section{Das Panorama der Arktis}

Dass Nordpolarexpeditionen im 19. und 20. Jahrhundert geradezu magnetisch die öffentliche Aufmerksamkeit anzogen, hing auch mit der besseren Bildung größerer Bevölkerungsschichten und der Verbreitung neuer Bildmedien zusammen. In einem aufwändig illustrierten Band Arctic Spectacles. The Frozen North in Visual Culture, 1818-1875 untersucht der amerikanische Literaturwissenschaftler und Arktisspezialist Russel A. Potter die Bedeutung von Panoramabildern, Laterna magica, Photographie und Stereoskopie für die Popularisierung der Arktisforschung. Die wachsende Faszination des Nordens ging einher mit der Verbreitung von Ausstellungen, Photographien und illustrierten Zeitschriften. Dabei begannen sich die Grenzen zwischen Kunst und Volkskultur, Wissenschaft, Kommerz und Unterhaltung aufzulösen: 
„Franklin sailed at the dawn of a new visual culture of mass history, mass images, and a mass reading public." (Potter, S. 72). Wer systematische Unterscheidungen zwischen den vielfältigen Medienformaten und Formen der Wissensrepräsentation erwartet, wird mit Potter nicht zufrieden sein. Hierzu ist jedoch gerade ein instruktiver Aufsatz von Dorit Müller (2009) erschienen, die am Beispiel der Geschichte der Polarforschung ausführlich auf die Charakteristika von Schriftsprache, Photographie und Film als Medienformaten der Wissenschaftspopularisierung eingeht.

Potter stellt dagegen die empirischen Fakten in den Mittelpunkt: Zwischen 1818 und 1883 existierten diesseits und jenseits des Atlantiks ganze 60 Arktisshows. Allein drei Panoramen mit Arktismotiven standen von 1819 bis 1850 am Londoner Leicester Square - mehr als von jeder anderen Weltregion. In großen Tourneen zogen die Schauen durch die Provinz und erreichten breite Bevölkerungsschichten und Berufsgruppen, sowohl Männer als auch Frauen. In den USA entstand 1857 auf der Grundlage des Reiseberichts von Elisha K. Kane das erste amerikanische Arktis-Panorama mit 57 Bildszenen. Bald darauf wurden zusätzlich auch Originalobjekte wie Kajaks, Walrosszähne und Narwalhörner einbezogen. Hatten die Expeditionen die Kenntnisse der Inuit zunächst kaum zu nutzen verstanden, führte deren wachsende Einbeziehung nun auch dazu, dass einige von ihnen mitgebracht wurden und in der staunenden Öffentlichkeit eine zweifelhafte Berühmtheit erlangten. Das Prinzip Veranschaulichung erlebte einen Höhepunkt, als die Überreste der katastrophal gescheiterten Expedition von John Franklin nach 14 Jahren gefunden und unter größtem Aufsehen in London ausgestellt wurden. Der Erfolg der Panoramen und Arktisshows ging erst mit der Erfindung des Kinos zu Ende. Zum Privatgebrauch wurden von nun an Dias und Stereoskope in Massen produziert.

Interessanterweise war es gerade John Franklin, der ein Exemplar des ersten Daguerreschen Photoapparats mit in die Arktis gebracht hatte. Potter berichtet, dass sich von den Aufnahmen nur die bei der Abreise aufgenommen Porträts der Expeditionsteilnehmer erhalten haben. Sie gehören zu den frühesten Photographien überhaupt und sollten die letzten Aufnahmen der Männer sein. Potters Band enthält vierzehn nach den Photos angefertigte Kupferstiche aus den Illustrated London News. Der Band verfügt außerdem über zahlreiche Abbildungen von stereoskopischen Photographien, Handzetteln, Kupferstichen und Zeitungsillustrationen. Im Anhang befindet sich ein chronologisches Verzeichnis aller Arktisshows zwischen 1819 und 1896.

\section{Öffentlichkeit als Argument}

Während Potters Band wesentlich von seinen Abbildungen und den faszinierenden historischen Medien profitiert, legte der amerikanische Jurist und Arktis-Sammler Douglas W. Wamsley im vergangenen Jahr das Ergebnis 
aufwändiger Archivarbeiten vor. Er schrieb die erste Biografie des amerikanischen Arztes und Polarforschers Isaac Israel Hayes (1832-1881), der als 21jähriger an Kanes Expedition teilnahm und in der zweiten Hälfte des 19. Jahrhunderts zu den erfolgreichsten Publizisten des Polarthemas gehörte, aber heute fast vergessen ist. Die detailreiche und mit knapp 600 Seiten umfangreiche Arbeit beruht auf der erstmaligen Auswertung von Quellen in lokalen Archiven, Manuskriptsammlungen und Briefwechseln der Familie. Die Erzählung folgt in großen Teilen der Lebensgeschichte von Hayes. Aufgewachsen in einer QuäkerFamilie in Pennsylvania brachte er sein Medizinstudium in zwei Jahren zu Ende, um sich für die Teilnahme an der Polarexpedition zu bewerben, deren Verlauf ausführlich geschildert wird. Der eigentliche Gewinn von Wamsleys Arbeit für die Popularisierungsgeschichte der Arktis aber liegt in dem umfassend ausgewerteten Material zur Geschichte eines Mediums, das sich parallel zur Popularisierung der Arktisfahrten schnell verbreitete: In den 1850er Jahren waren populärwissenschaftliche Vorträge sehr beliebt.

Durch die neuen Verkehrsmittel Eisenbahn und Telegraphie war es möglich, dass Redner durch das Land reisten und auch abseits der Metropolen praktisches Grundlagenwissen und nicht zuletzt moralische Orientierung vermittelten. Solche öffentlichen Vorträge waren das bevorzugte Mittel von Isaac I. Hayes, um wissenschaftliche Anerkennung zu gewinnen, die Finanzierung seiner Expeditionen und schließlich auch seinen Lebensunterhalt zu verdienen. In Vorträgen vor der American Geographical Society in New York und in der Smithsonian Institution in Washington D.C. sprach Hayes 1857 und 1858 über das offene Polarmeer. Seine Theorie stieß auf große Aufmerksamkeit und wurde mittels der neuen Tageszeitungen weit verbreitet. Er gewann die Unterstützung einflussreicher wissenschaftlicher Gesellschaften, hatte aber ohne staatliche Unterstützung Probleme, die Expedition zu finanzieren. Erst 1860 konnte er aufbrechen und erreichte ein Jahr später scheinbar das Ziel, als er am nördlichsten Punkt der Reise eisfreie Gewässer vor sich zu sehen glaubte. Dem Reisebericht gab er den windigen Titel The Open Polar Sea und konnte damit den publizistischen Erfolg von Kane wiederholen. Auch Hayes Vorträge waren gut besucht, weil sie den Horizont in Richtung Norden öffneten und Hoffnung und Nationalstolz vermittelten. Doch das wissenschaftliche Ergebnis war begrenzt. Das größte Interesse fand Hayes, der bis zu seinem Tod an einen eisfreien Nordpol glaubte, bei dem deutschen Geographen August Petermann in Gotha, der zwei deutsche Polarexpeditionen initiierte, ohne jemals selbst in der Arktis gewesen zu sein (siehe dazu jetzt Felsch 2010).

\section{Ein britisches Epos}

Die europäische Rezeption der Arktisforschung verlief nicht wesentlich anders als in den Vereinigen Staaten. Die jahrelange Suche nach John Franklin hat das 
Interesse wachgehalten. Sie bildet deshalb den Ausgangs- und Zielpunkt der 2008 veröffentlichten literaturwissenschaftlichen Doktorarbeit von Janice Cavell, Tracing the Connected Narrative. Arctic Exploration in British Print Culture, 1818-1860. Die Hauptquellen für ihre Untersuchung liefern Berichte in populären Zeitschriften und Tageszeitungen. Über die Jahre wurde die Kette der Misserfolge von Arktisexpeditionen zu einer Art publizistischer Fortsetzungsgeschichte, in der sich Reiseberichte, Briefe, Vortragsmanuskripte und Buchrezensionen abwechselten. Cavell analysiert, wer in welcher Zeitschrift für welches Publikum geschrieben hat und stellt die Frage, wie Zeitgenossen das Schicksal Franklins und der früheren Arktisexpeditionen beurteilt haben.

Viele Forscher und ihre Angehörigen, Journalisten und Rezensenten teilten dabei nicht nur gesellschaftliche Stereotype mit, sondern auch persönliche Einschätzungen, die Mentalitäten und persönliche Überzeugungen erkennen lassen. Die ideologischen Auseinandersetzungen zwischen Tories, Whigs und Radikalen wurden - wie Cavell behauptet - auch auf den Seiten der Quarterly Review, der Edinburgh Review oder des Examiner ausgefochten. Cavell zieht daraus Rückschlüsse auf kulturelle und politische Überzeugungen, die über die Ergebnisse der Studie von Riffenburgh (1993) weit hinausgehen. John Barrow hatte etwa in der konservativen Quarterly Review die Vision einer religiös verankerten Nation entwickelt, die sich in der Arktis gegen sozialrevolutionäre Herausforderungen behauptete. Christliche Zeitungen wie der Christian Observer, die British Review und die London Critical Review schlossen, dass die göttliche Vorsehung das britische Volk ausersehen hätte. Doch nach 1848 hatten sich die politischen Verhältnisse in Europa derart verändert, dass sich nun auch einzelne Personen wie Franklins Witwe Jane gegen die Regierung stellen und in Eigeninitiative Schiffe zum Nordpol ausrüsten konnten. Allein die Times betrachtete Arktisreisen von vornherein als Geldverschwendung.

Cavell bestätigt nicht nur, dass sich das britische Selbstverständnis als Seeund Kolonialmacht auch in der Arktis manifestierte, sondern sie entdeckt ebenso romantische Motive im britischen Imperialismus. Nur eine ritterliche Anerkennung der Übermacht des Eises und die demütige Unterordnung unter seine Gesetze, nicht englisches Selbstbewusstsein und viktorianisches Fortschrittsvertrauen, sondern Bescheidenheit und Selbstbeschränkung waren charakterliche Ideale, mit denen die Erforschung der Arktis gelingen konnte. Cavells Arbeit beginnt und endet mit der Nachricht vom Tod Franklins. Seine Tagebücher zeigen, wie er angesichts der großen Strapazen tief religiös wurde. Später gewann er dadurch in der britischen Öffentlichkeit den Charakter eines moralischen Vorbilds. Als erste Berichte davon sprachen, dass es unter seinen Kameraden zu Kannibalismus gekommen sein könnte, wurden die Überbringer der Nachricht offen kritisiert. Für Cavell konnte erst Leopold McClintock das Epos der britischen Arktiserforschung zu Ende 
bringen, als er Franklin nach dem Auffinden der Überreste seiner Expedition von diesem Verdacht freisprach. Stattdessen hob er dessen Leistungen hervor: die Entdeckung der Nordwest-Passage und der christliche Geist, in dem er und seine Männer ihr tragisches Schicksal ertragen hätten. Ihre Männlichkeit hing mehr mit ihrem Charakter als mit körperlicher Kraft zusammen und kannte auch schwache Seiten: Geduld und Ausdauer, Abwarten und Erdulden waren in der Arktis lebenswichtige Qualitäten.

\section{Weiße Flecken}

Den epischen Charakter der Arktisforschung nimmt auch Marion Munz-Krines zum Ausgangspunkt ihrer 2009 erschienenen Promotion mit dem Titel Expeditionen ins Eis. Historische Polarreisen in der Literatur. Sie untersucht anhand literarischer Quellen, wie sich der historische Typus der Polarforscher seit dem 19. Jahrhundert verändert hat und welche Rolle die Öffentlichkeit bei ihrer Stilisierung zu Nationalhelden gespielt hat. Allerdings hat die Arbeit nicht die Gelegenheit genutzt, die historischen Imaginationen der Arktis literaturwissenschaftlich genauer zu untersuchen und für die Wissensgeschichte fruchtbar zu machen. Denn historische Analysen des anhaltenden Arctic Fever, das zeigen alle besprochenen Arbeiten, ermöglichen besonders produktive Querverbindungen zwischen Medien-, Wissenschafts- und Kulturgeschichte.

Dabei sind diese Studien selbst Teil der von ihnen beschriebenen Konjunktur. Michael Robinson hat die von ihm erkannte Gunst der Stunde zusätzlich genutzt, indem er aus Anlass des Internationalen Polarjahres die Ausstellung The Coldest Crucible. Arctic Exploration in American Culture kuratiert hat. Die Ausstellung wurde vom 14. März bis 21. Juni 2009 im Portland Museum of Art im US-Bundesstaat Maine gezeigt. Zu den Exponaten gehörten Karten, Bücher und Grafiken aus der Sammlung der Osher Map Library an der University of Southern Maine in Portland, darunter Arktiskarten der Renaissance, Arbeiten der Arktisillustratoren Frederick Church und William Bradford, sowie Photographien und Erinnerungsstücke von Robert Peary. Ergänzt durch 19 zeitgenössische künstlerische Arbeiten hat Robinson die Arktisforscher hier wiederum aus dem ewigen Eis in die Mitte der amerikanischen Gesellschaft zurückgeholt.

Arktisforschung steht heute meist im Zeichen der Klimaforschung. Öffentliche Spekulationen über einen eisfreien Nordpol haben dabei eine lange Tradition. Als John Barrow 1818 in der Quarterly Review von eisfreien Gewässern am Pol berichtete, verkaufte sich die entsprechende Ausgabe alleine am Erscheinungstag über 12.000 Mal. Elisha K. Kane, Isaac I. Hayes und August Petermann popularisierten die Vorstellung eines eisfreien Polarmeeres. Es geriet erst in Vergessenheit, als immer mehr Polarforscher dieses gegen Ende des 19. Jahrhunderts in das Reich der Mythen 
verabschiedeten. Hielt der Walfänger und Gelehrte William Scoresby es schon damals für möglich, dass ein Rückgang des Eises durch einen Anstieg der Temperaturen ausgelöst werde (Cavell 2008: 62), so ist dieser Zusammenhang inzwischen Realität geworden. Wir wissen längst davon, denn die Betriebstemperatur der Arktis und ihrer Erforschung wird weiterhin unter größter öffentlicher Anteilnahme gemessen.

\section{Danksagung}

Der Beitrag wurde durch ein Fellowship der Smithsonian Institution, Washington, D.C., ermöglicht.

\section{Literatur}

Borck, Cornelius, 2009. Bild der Wissenschaft. Neuere Sammelbände zum Thema Visualisierung und Öffentlichkeit. NTM. Zeitschrift für Geschichte der Wissenschaften, Technik und Medizin, 17, 317-327.

David, Robert, 2000. The Arctic in the British Imagination 1818-1914. Manchester/New York: Manchester University Press.

Felsch, Philipp, 2009. Der Traum vom Nordpol. Robert Peary, Frederick Cook - und ein deutscher Kartograf. Neue Zürcher Zeitung, 11. April 2009.

Felsch, Philipp, 2010. Wie August Petermann den Nordpol erfand. München: Luchterhand.

McGoogan, Ken, 2008. Race to the Polar Sea. The Heroic Adventures of Elisha Kent Kane. Berkeley, CA: Counterpoint Press.

Müller, Dorit, 2009. Transformationen populären Wissens im Medienwandel am Beispiel der Polarforschung. In: Petra Boden und Dorit Müller, Hg., Populäres Wissen im medialen Wandel seit 1850, Berlin: Kadmos, 35-79.

Munz-Krines, Marion, 2009. Expeditionen ins Eis. Historische Polarreisen in der Literatur, Frankfurt am Main: Peter Lang.

Oeser, Erhard, 2008. Die Jagd zum Nordpol. Tragik und Wahnsinn der Polarforscher, Darmstadt: Wissenschaftliche Buchgesellschaft.

Riffenburgh, Beau, 1993. The Myth of the Explorer. The Press, Sensationalism, and Geographical Discovery, London: Belhaven Press.

Sawin, Mark Metzler, 2009. Raising Kane: Elisha Kent Kane and the Culture of Frame in Antebellum America, Philadelphia: American Philosophical Society Press.

Spufford, Francis, 1997. I May be Some Time. Ice and the English Imagination, New York: Picador.

Zeilinger, Johannes, 2009. Auf brüchigem Eis. Frederick A. Cook und die Eroberung des Nordpols, Berlin: Matthes \& Seitz.

Christian Holtorf

Stiftung Deutsches Hygiene-Museum

Abteilung Wissenschaft

Lingnerplatz 1

01069 Dresden

Deutschland

E-Mail: christianholtorf@hotmail.com 